\title{
Structural insights into the ferroxidase and iron sequestration mechanisms of ferritin from Caenorhabditis elegans
}

\author{
T.R. Malcolm¹, S. Mohamed Murbarak ${ }^{1}$, E. Hanssen ${ }^{2}$, H.G. Brown ${ }^{2}$, G. McColl ${ }^{3}$, M.J. Maher ${ }^{1,4}$ \& G.N.L. Jameson ${ }^{1}$ \\ ${ }^{1}$ School of Chemistry, Bio21 Molecular Science and Biotechnology Institute, The University of Melbourne, Parkville, Victoria, \\ Australia. \\ ${ }^{2}$ Ian Holmes Imaging Centre, Bio21 Molecular Science and Biotechnology Institute, University of Melbourne, Parkville, Victoria, \\ Australia. \\ ${ }^{3}$ The Florey Institute of Neuroscience and Mental Health, The University of Melbourne, Parkville, Victoria, Australia. \\ ${ }^{4}$ Department of Biochemistry and Genetics, La Trobe Institute for Molecular Science, La Trobe University, Melbourne, Victoria, \\ Australia. \\ tess.malcolm@unimelb.edu.au
}

Iron is an essential trace element required for a multitude of cellular processes [1]. When in excess iron becomes toxic, therefore its intracellular concentration must be strictly regulated by a number of interacting mechanisms [2]. Ferritin is a ubiquitous iron-storage protein that forms a highly conserved 24-subunit spherical cage-like structure. Ferritin catalyses the oxidation of iron (II) to iron (III) by dioxygen at a di-iron site called the ferroxidase centre, and the newly oxidised iron (III) is then sequestered as a mineral core to prevent cellular damage [3]. As part of a greater study to understand iron transport we utilise the model organism, Caenorhabditis elegans, to investigate and elucidate these processes.

C. elegans contains two ferritin proteins, FTN-1 and FTN-2, that are orthologous to the human ferritins [4]. FTN-1 and FTN-2 both exhibit ferroxidase activity, although FTN-2 catalyses the oxidation of iron (II) at a rate significantly faster than FTN-1. All residues involved in catalysis are conserved between FTN-1 and FTN-2, suggesting that these mechanistic anomalies are due to structural differences at a location distinct to the ferroxidase centre. To address this, we solved the structures of both FTN-1 and FTN-2 by X-ray crystallography to $1.84 \AA$ and $1.47 \AA$ resolution respectively, and the structure of FTN-2 by cryo electron microscopy to $1.88 \AA$. FTN1 and FTN-2 both adopt the conserved 24-subunit cage-like structure and bind one metal in the higher affinity "A site" of the di-iron ferroxidase centre of each chain [3]. Further comparative analyses using both X-ray crystallography and electron microscopy techniques, reveal the structural features that influence iron influx, catalysis and transfer to the mineral core.

These structural insights will further our understanding of the mechanisms that ferritin utilizes to regulate iron storage and its role in the iron homeostasis. These findings will have further implications for diagnosis and treatment of haemochromatosis, anaemia and other iron related diseases.

[1] Anderson, G.J. \& Frazer, D.M. (2017). Am. J. Clin. Nutr. 106, 1559S-1566S.

[2] Aisen, P., Enns, C. \& Wessling-Resnick, M. (2001). Int. J. Biochem. Cell Biol. 33 (10), 940-959.

[3] Ebrahimi, K.H., Hagedoorn, P. \& Hagen, W.R. (2015). Chem Rev. 115 (1), 295-326.

[4] Anderson, C.P. \& Leibold, E.A. (2014). Front Pharmacol. 5 (113).

Keywords: Iron homeostasis; ferritin; ferroxidase; Caenorhabditis elegans; X-ray crystallography, Cryo electron microscopy

This work was supported by the ARC grant DP200100110 to G.N.L. Jameson and G. McColl. 